\title{
A study of the product distribution in the epoxidation of propylene over
}

TS-1 catalyst in a trickle bed reactor

\author{
Matias Alvear, Kari Eränen, Dmitry Yu. Murzin, Tapio Salmi ${ }^{\star}$
}

Laboratory of Industrial Chemistry and Reaction Engineering, Johan Gadolin

Process Chemistry Centre (PCC), Åbo Akademi University, 20500 Turku/Åbo

Finland

tapio.salmi@abo.fi

\section{Supplementary information}

\section{Conversion, selectivity and carbon balance}

The carbon balance was defined as

$$
n_{\text {out }}=n_{C_{3} H_{6}}^{G a S}+n_{C_{3} H_{6} O}^{G a s}+n_{C_{3} H_{6} O}^{\text {Liquid }}+n_{C_{4} H_{10} O_{2}}^{\text {Liquid }}
$$

where $n_{i}$ are the mol in the out stream of each product in liquid or gas phase. The carbon balance exhibited be between $97 \%$ and $103 \%$ of the initial moles of reactants fed into the reactor.

The propylene conversion $(X)$ was defined as

$$
X=\frac{\dot{n}_{\text {in }}-\dot{n}_{\text {out }}}{\dot{n}_{\text {in }}}=\frac{c_{\text {in }} \dot{V}_{\text {in }}-c_{\text {out }} \dot{V}_{\text {out }}}{c_{\text {in }} \dot{V}_{\text {in }}}
$$

where $c_{\text {in }}$ and $c_{\text {out }}$ are the inlet and outlet concentrations, while $\dot{V}_{\text {in }}$ and $\dot{V}_{\text {out }}$ are the inlet and outlet volumetric flows, respectively.

The selectivity was calculated as 


$$
\text { Selectivity }(\%)=\frac{\text { product flow }\left[\frac{\mathrm{mol}}{\mathrm{min}}\right]}{\text { converted propylene }\left[\frac{\mathrm{mol}}{\mathrm{min}}\right]} * 100 \%
$$

\section{Experimental matrix}

Table S.1 Experimental matrix

\begin{tabular}{|c|c|c|c|c|c|c|c|c|}
\hline & Run & $\mathrm{T}\left({ }^{\circ} \mathrm{C}\right)$ & $P($ bar $)$ & $\begin{array}{l}\text { Propylene } \\
\text { flow } \\
\text { (mL/min) }\end{array}$ & $\begin{array}{c}\mathrm{H}_{2} \mathrm{O}_{2} \text { flow } \\
(\mathrm{mL} / \mathrm{min})\end{array}$ & $\begin{array}{c}\mathrm{H}_{2} \mathrm{O}_{2} \\
(\mathrm{wt} \%)\end{array}$ & $\begin{array}{l}\mathrm{H}_{2} \mathrm{O} \\
\text { (wt\%) }\end{array}$ & $\begin{array}{c}\text { Methanol } \\
\text { (wt\%) }\end{array}$ \\
\hline & \multicolumn{8}{|c|}{ Experimental set up } \\
\hline \multirow{4}{*}{ A } & 1 & 25 & 4.5 & 5 & 0.5 & 1 & 2 & 97 \\
\hline & 2 & 30 & 4.5 & 5 & 0.5 & 1 & 2 & 97 \\
\hline & 3 & 35 & 4.5 & 5 & 0.5 & 1 & 2 & 97 \\
\hline & 4 & 25 & 4.5 & 5 & 0.5 & 1 & 2 & 97 \\
\hline \multirow{4}{*}{ B } & 5 & 25 & 4.5 & 7.5 & 0.5 & 1 & 2 & 97 \\
\hline & 6 & 30 & 4.5 & 7.5 & 0.5 & 1 & 2 & 97 \\
\hline & 7 & 35 & 4.5 & 7.5 & 0.5 & 1 & 2 & 97 \\
\hline & 8 & 25 & 4.5 & 7.5 & 0.5 & 1 & 2 & 97 \\
\hline \multirow{4}{*}{ C } & 9 & 25 & 4.5 & 5 & 0.5 & 2 & 5 & 93 \\
\hline & 10 & 30 & 4.5 & 5 & 0.5 & 2 & 5 & 93 \\
\hline & 11 & 35 & 4.5 & 5 & 0.5 & 2 & 5 & 93 \\
\hline & 12 & 25 & 4.5 & 5 & 0.5 & 2 & 5 & 93 \\
\hline \multirow{4}{*}{ D } & 13 & 25 & 4.5 & 5 & 0.5 & 4 & 9 & 87 \\
\hline & 14 & 30 & 4.5 & 5 & 0.5 & 4 & 9 & 87 \\
\hline & 15 & 35 & 4.5 & 5 & 0.5 & 4 & 9 & 87 \\
\hline & 16 & 25 & 4.5 & 5 & 0.5 & 4 & 9 & 87 \\
\hline \multirow{3}{*}{$\mathrm{E}$} & 17 & 25 & 2.5 & 5 & 0.5 & 2 & 5 & 93 \\
\hline & 18 & 25 & 4.5 & 5 & 0.5 & 2 & 5 & 93 \\
\hline & 19 & 25 & 6.5 & 5 & 0.5 & 2 & 5 & 93 \\
\hline
\end{tabular}




\begin{tabular}{|c|c|c|c|c|c|c|c|c|}
\hline & 20 & 25 & 8.5 & 5 & 0.5 & 2 & 5 & 93 \\
\hline & 21 & 25 & 2.5 & 5 & 0.5 & 2 & 5 & 93 \\
\hline \multirow{5}{*}{$F$} & 22 & 35 & 4.5 & 5 & 0.25 & 4 & 9 & 87 \\
\hline & 23 & 35 & 4.5 & 5 & 0.5 & 4 & 9 & 87 \\
\hline & 24 & 35 & 4.5 & 5 & 1 & 4 & 9 & 87 \\
\hline & 25 & 35 & 4.5 & 5 & 2 & 4 & 9 & 87 \\
\hline & 26 & 35 & 4.5 & 5 & 0.25 & 4 & 9 & 87 \\
\hline \multirow{6}{*}{ G } & 27 & 25 & 8.5 & 5 & 0.5 & 2 & 5 & 93 \\
\hline & 28 & 40 & 8.5 & 5 & 0.5 & 2 & 5 & 93 \\
\hline & 29 & 50 & 8.5 & 5 & 0.5 & 2 & 5 & 93 \\
\hline & 30 & 60 & 8.5 & 5 & 0.5 & 2 & 5 & 93 \\
\hline & 31 & 80 & 8.5 & 5 & 0.5 & 2 & 5 & 93 \\
\hline & 32 & 25 & 8.5 & 5 & 0.5 & 2 & 5 & 93 \\
\hline \multirow{5}{*}{$\mathrm{H}$} & 33 & 60 & 8.5 & 5 & 0.5 & 2 & 20 & 78 \\
\hline & 34 & 60 & 8.5 & 5 & 0.5 & 2 & 30 & 68 \\
\hline & 35 & 60 & 8.5 & 5 & 0.5 & 2 & 40 & 58 \\
\hline & 36 & 60 & 8.5 & 5 & 0.5 & 2 & 20 & 78 \\
\hline & 37 & 60 & 8.5 & 5 & 0.5 & 2 & 20 & 78 \\
\hline
\end{tabular}

\section{Evaluation of flow pattern}

The dimensionless parameter $F=w_{L} \rho_{L} /\left(w_{G} \rho_{G}\right)$ ( $w=$ velocity, $\rho=$ density) was calculated for the equal gas molar flows of propylene and inert gas (helium) for different total liquid flows (see the table below) through the reactor tube. Because of the low total pressure ideal gas law was applied,

Liquid flow $/(\mathrm{ml} / \mathrm{min}) \quad F$

0.25

24.7

1.0

98.8

2

198

Parameter $\mathrm{w}_{\mathrm{G}} \rho_{\mathrm{G}}$ was estimated to $0.00381 \mathrm{~kg} /\left(\mathrm{m}^{2} \mathrm{~s}\right)$. According to the flow charts published for three-phase packed beds (Salmi, Mikkola Wärnå, Chemical Reaction Engineering and Reactor Technology, CRC Press 2019), these values ( $w_{G} \rho_{G}$ and $F$ ) are within the trickle flow domain. 